\title{
IncRNA UASR1 sponges miR-107 in colorectal cancer to upregulate oncogenic CDK8 and promote cell proliferation
}

\author{
QIZHI ZHANG ${ }^{1}$ and ZHAOSHENG CHEN ${ }^{2}$ \\ ${ }^{1}$ Outpatient Department and ${ }^{2}$ Department of Gastroenterology, \\ The Second Hospital of Shandong University, Jinan, Shandong 250033, P.R. China
}

Received April 20, 2020; Accepted August 19, 2020

DOI: $10.3892 / \mathrm{ol} .2020 .12168$

\begin{abstract}
IncRNA UASR1 (UASR1) has been characterized as an oncogenic lncRNA in breast cancer. UASR1 was predicted to interact with miR-107, which serves tumor suppressive roles mainly by targeting CDK8. The present study was performed to investigate the interactions among UASR1, miR-107 and CDK8 in colorectal cancer (CRC). A total of 62 patients with $\mathrm{CRC}$, including 40 males and 22 females (age range, 38-67 years; mean age, 57.2 \pm 7.6 years) were enrolled at the Second Hospital of Shandong University between July 2012 and July 2014. The expression of UASR1 in tissues and cells were detected by reverse transcription-quantitative polymerase chain reaction. The interaction between UASR 1 and miR-107 was investigated by performing dual luciferase activity assay, and the effects of overexpression of UASR1, miR-107 and CDK8 on the proliferation of CR4 cells were analyzed by performing cell proliferation analysis. It was observed that UASR1 is upregulated in CRC and its high expression levels predicted poor survival in patients with CRC. RNA-RNA interaction prediction demonstrated that UASR1 may interact with miR-107. In CRC cells, overexpression of UASR1 and miR-107 did not affect each other. However, the expression of CDK8, a target of miR-107, was upregulated following overexpression of UASR1. Notably, overexpression of UASR 1 decreased the inhibitory effects of miR-107 on cell proliferation and the expression of CDK8. Therefore, UASR1 may sponge miR-107 to upregulate oncogenic CDK8, thereby promoting CRC cell proliferation.
\end{abstract}

\section{Introduction}

Colorectal cancer (CRC) is a common malignancy in males and females worldwide (1). According to the latest GLOBOCAN data, CRC caused 551,269 mortalities, accounting for 5.8\% of

Correspondence to: Dr Zhaosheng Chen, Department of Gastroenterology, The Second Hospital of Shandong University, 247 Beiyuan Street, Jinan, Shandong 250033, P.R. China

E-mail: aq1363@163.com

Key words: UASR1, colorectal cancer, miR-107, CDK8, proliferation all cancer-associated mortalities in 2018 (2). In the same year, a total of 1,096,601 new cases of CRC were diagnosed, accounting for $6.1 \%$ of all new cancer cases (2). Survival of patients with CRC is significantly affected by cancer stages $(3,4)$. More than $90 \%$ of patients with localized CRC can live longer than 5 years after initial diagnosis, while this percentage may drop to $14 \%$ once distant tumor metastasis occurred $(3,4)$. Therefore, early diagnosis remains the key for the survival of patients with CRC $(5,6)$. However, sensitive early diagnostic biomarkers for CRC are lacking and patient with CRC are mostly diagnosed at advanced stages.

Molecular signals serve critical roles in the occurrence and development of CRC $(7,8)$. Functional analyses of molecular pathways involved in CRC provide guidance for the development of anti-CRC therapy with novel targets (9). It has been well established that long non-coding RNAs (lncRNAs, >200 nt) encode no proteins but participate in human disease by regulating disease-related gene expression $(10,11)$. Therefore, regulating the expression of certain IncRNAs may contribute toward the recovery of patients with cancer (12). However, the functions of most lncRNAs remain elusive. IncRNA UASR1 (UASR1) has been characterized as an oncogenic lncRNA in breast cancer (13). In a recent study, UASR1 was reported to promote the proliferation of CRC cells by interacting with the mTOR pathway (14). However, the mechanism of the function of UASR1 in CRC is yet to be fully elucidated. We hypothesized that UASR1 may interact with miR-107, which serves tumor suppressive roles mainly by targeting CDK8 (15). The present study was therefore performed to investigate the interactions among UASR1, miR-107 and CDK8 in CRC.

\section{Materials and methods}

CRC patients and tissue specimens. The present study was approved by the Ethics Committee of the Second Hospital of Shandong University (no. CRC201233255). A total of 62 patients with $\mathrm{CRC}$, including 40 males and 22 females (age range, 38-67 years; mean age, 57.2 \pm 7.6 years) were enrolled at the aforementioned hospital between July 2012 and July 2014. All patients were diagnosed with CRC by histopathological examination. All patients were newly diagnosed cases and other clinical disorders were excluded. Therapy was not innititaed prior to this study. All patients provided written informed consent. Prior to therapy, biopsy was performed on 62 patients 
with CRC to collect CRC tumor tissues and paired non-tumor tissue samples.

Treatment and follow-up. The 62 patients were staged according to American Joint Committee on Cancer staging criteria (16). There were $12,17,15$ and 18 cases at stage I, II, III and IV, respectively. Patients were treated with chemotherapy, surgical resection, radiotherapy or immunotherapy according to patients' clinical stages or health conditions. All patients were followed up monthly for 5 years from the day of admission to record patients survival. All 62 patients conpleted the follow-up study.

CRC cells and transfections. Human CRC cell line CR4 (Sigma-Aldrich; Merck KGaA) was used. Cell culture medium was composed of fetal bovine serum (FBS; 10\%; Sigma-Aldrich; Merck KGaA) and Eagle's minimal essential medium (EMEM; 90\%; Sigma-Aldrich; Merck KGaA). Cell culture conditions were $95 \%$ humidity, $37^{\circ} \mathrm{C}$ and $5 \% \mathrm{CO}_{2}$. Cells were harvested at $\sim 85 \%$ confluence to perform subsequent experiments. With the pcDNA3.1 vector (Invitrogen; Thermo Fisher Scientific, Inc.) as a backbone, expression vectors of UASR1 and CDK8 were constructed. Negative control (NC) miRNA (5'-UGUAACGUACGUUCGUACCGUGA-3') and miR-107-mimic (5'-AGCAGCAUUGUACAGGGCUAU CA-3') were purchased from Sigma-Aldrich (Merck KGaA). CR4 cells $\left(10^{7}\right.$ cells in $10 \mathrm{ml}$ medium) were transfected with $10 \mathrm{nM}$ vector and/or $40 \mathrm{nM}$ miRNA using Lipofectamine 2000 (Invitrogen; Thermo Fisher Scientific, Inc.). In all transfections, untransfected cells were used as control (C) cells and NC miRNA- or empty vector-transfected cells were used as $\mathrm{NC}$ cells. Cells were collected at $48 \mathrm{~h}$ post-transfection prior to subsequent experiments. In cases of co-transfection, cells were simultaneously transfected with UASR 1 expression vector and miR-107.

Dual luciferase activity assay. With the pGL3 vector (Promega Corporation) as the backbone, a luciferase vector of UASR1 was constructed. The interaction between UASR 1 and miR-107 was investigated by performing a dual luciferase activity assay, which was performed by co-transfecting CR4 cells with UASR1 luciferase vector + miR-107 mimic (miR-107 group) or UASR1 luciferase vector + NC miRNA (NC group). Dual-luciferase reporter assay system (Promega Corporation) was used for the determination of relative luciferase activities at $48 \mathrm{~h}$ post-transfection. Firefly luciferase activity was normalized to Renilla luciferase activity. The value of the NC group was set to ' 1 ', and value of miR-107 group was normalized to NC group.

RNA preparations. Total RNA was isolated from CR4 cells and paired tissue samples using TRIzol reagent (Invitrogen; Thermo Fisher Scientific, Inc.). To harvest miRNAs, $85 \%$ ethanol was used to precipitate and wash RNA samples. Genomic DNAs in RNA samples were removed by digesting with gDNA eraser (Takara Biotechnology Co., Ltd.).

Reverse transcription-quantitative polymerase reaction (RT-qPCR). Reverse transcription $\left(55^{\circ} \mathrm{C}\right.$ for $20 \mathrm{~min}$ and $85^{\circ} \mathrm{C}$ for $10 \mathrm{~min}$ ) was performed to synthesize cDNA using SuperScriptIV
Reverse Transcriptase (Invitrogen; Thermo Fisher Scientific, Inc.). With cDNA samples as the template, qPCR reactions were prepared using QuantiTect SYBR Green PCR kit (Qiagen $\mathrm{GmbH}$ ) to determine the expression of UASR1 and CDK8 mRNA with GAPDH as the internal control. Poly (A) addition, mature miRNA reverse transcription and qPCR reactions were performed using an All-in-One ${ }^{\mathrm{TM}}$ miRNA RT-qPCR Detection kit (GeneCopoeia, Inc.) to measure the expression levels of mature miR-107. Three replicate reactions were included in each experiment and data were normalized using $2^{-\Delta \Delta \mathrm{Cq}}$ method (17). The sample with the highest $\Delta \mathrm{Cq}$ value was set to ' 1 ', and all other samples were normalized to this group. Primer sequences were: UASR1 forward, 5'-GCG GATCGCAGACCCTAA-3' and reverse, 5'-AGAACACTT TGCGGAAGGC-3'; CDK8 forward, 5'-GAATTTCTATGT CGGCATGC-3' and reverse, 5'-ATAGTCAAAGAGAAGCCA TACTTT-3'; and GAPDH forward, 5'-GTCTCCTCTGACTTC AACAGC-3' and reverse, 5'-CCACCCTGTTGCTGTAGC CAA-3'. The forward primer for miR-107 was 5'-AGCAGC ATTGTACAGGGCTATCA-3'. The universal reverse primer and U6 forward primer were from the kit. PCR reaction cycles were as follows: $95^{\circ} \mathrm{C}$ for $1 \mathrm{~min}$, followed by 40 cycles of $95^{\circ} \mathrm{C}$ for $10 \mathrm{sec}$ and $58^{\circ} \mathrm{C}$ for $40 \mathrm{sec}$.

Western-blot analysis. Total protein was isolated from CR4 cells using RIPA buffer (Invitrogen; Thermo Fisher Scientific, Inc.). After that, BCA assay (Invitrogen; Thermo Fisher Scientific, Inc.) was used to determine protein concentration, followed by denaturation in boiling water for $10 \mathrm{~min}$. Denatured protein samples (30 $\mu$ g per lane) were separated using $8 \%$ SDS-PAGE gel. Polyvinylidene difluoride membranes were used for gel transfer. Following blocking in PBS (5\% skimmed milk) at room temperature for $20 \mathrm{~min}$, membranes were first incubated with rabbit primary antibodies against CDK8 (1:1,000; cat. no. ab115155; Abcam) and GAPDH (1:1,000; cat. no. ab9485; Abcam) at $4^{\circ} \mathrm{C}$ for $12 \mathrm{~h}$, followed by incubation with an immunoglobulin $\mathrm{G}$ horseradish peroxidase-conjugated secondary antibody (1:1,000; cat. no. ab6721; Abcam) at room temperature for $2 \mathrm{~h}$. Signals were developed using enhanced chemiluminescent (Invitrogen; Thermo Fisher Scientific, Inc.). Image J 1.48 software (National Institutes of Health) was used to normalize signals. The value of $\mathrm{C}$ group was set to ' 1 ', and values of other groups were normalized to $\mathrm{C}$ group.

Cell proliferation assay. CR4 cells were harvested at $48 \mathrm{~h}$ post-transfection to perform a cell proliferation assay. In brief, 6,000 cells in $0.1 \mathrm{ml}$ medium were transferred to each well of a 96 -well plate. Cells were cultivated at $37^{\circ} \mathrm{C}$, followed by measurement of OD values at $450 \mathrm{~nm}$ every $24 \mathrm{~h}$ for a total of 96 h. CCK solution (Sigma-Aldrich; Merck KGaA) was added into each well at $4 \mathrm{~h}$ before the measurement of OD values. The value of the $\mathrm{C}$ group at $96 \mathrm{~h}$ was set to '100', and all other groups were normalized to the $\mathrm{C}$ group.

Statistical analyses. Each experiment was performed in three biological replicates. Data are expressed as the mean values. Comparison between paired tissues was performed using a paired t-test. An unpaired t-test was used to compare two independent groups. Analysis of variance, followed by Tukey's post hoc test was used to compare differences among multiple 
A

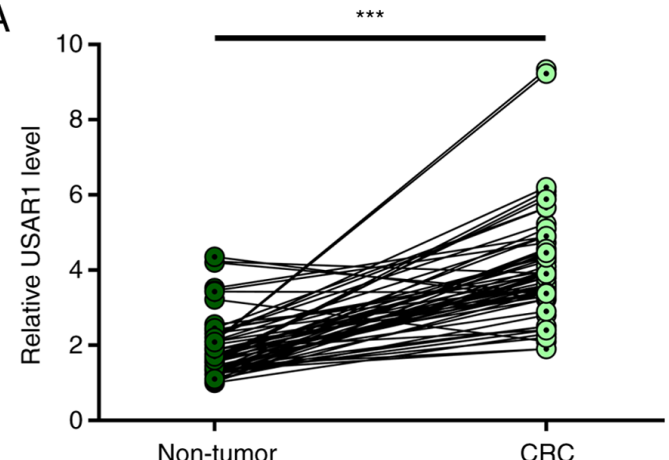

B

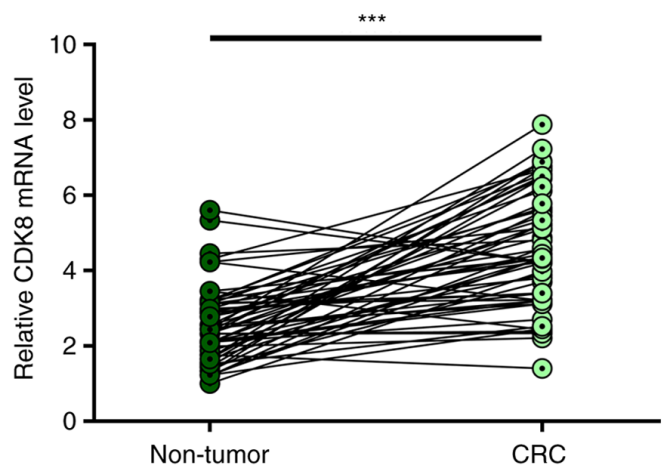

C

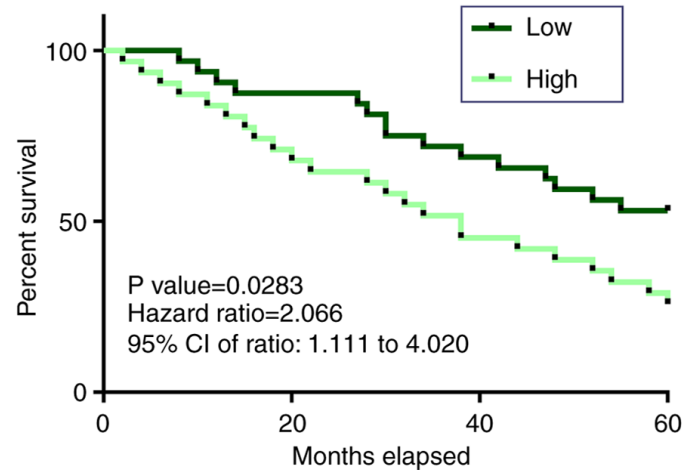

Figure 1. UASR1 and CDK8 were upregulated in CRC and high expression levels of UASR1 in CRC predicted poor survival. The expression of (A) UASR1 and (B) CDK8 in paired CRC and non-tumor tissues from 62 patients with CRC were determined by reverse transcription-quantitative polymerase chain reaction. ${ }^{* * *} \mathrm{P}<0.001$. The 62 patients with CRC were divided into high and low UASR1 expression groups $(\mathrm{n}=31)$ with the median level of UASR1 in CRC tissues as the cut-off value. Survival curves were plotted for the two groups based on follow-up data. Survival curves were compared using the log-rank test. (C) It is worth noting that miR-107 expression was not significantly correlated with patient survival ( $\mathrm{P}=0.488 ; \mathrm{HR}=1.123$; 95\% CI: 0.598-2.117), while the expression of $\mathrm{CDK} 8$ was correlated with patient survival ( $\mathrm{P}=0.0167 ; \mathrm{HR}=2.376 ; 95 \% \mathrm{CI}$ : 1.237-4.417). $\mathrm{CRC}$, colorectal cancer; $\mathrm{HR}$, hazards ratio; $\mathrm{CI}$, confidence interval.

groups. The 62 patients with CRC were divided into high and low UASR1 level groups $(\mathrm{n}=31)$ with the median level of UASR1 in CRC tissues as the cut-off value. Survival curves were plotted for the two groups based on follow-up data. Survival curves were compared using a log-rank test. $\mathrm{P}<0.05$ was considered to indicate a statistically significant difference.

\section{Results}

UASR1 and CDK8 were upregulated in CRC and high expression levels of UASRI in CRC predicted poor survival. Expression of UASR1 and CDK8 in paired non-tumor and CRC tissues from 62 patients with CRC were analyzed by performing RT-qPCR. Compared with non-tumor tissues, the expression levels of UASR1 (Fig. 1A) and CDK8 (Fig. 1B) were significantly higher in CRC tissues $(\mathrm{P}<0.001)$. Survival curves were plotted for both high and low UASR1 expression groups. Compared with patients in the low expression group, patients in the high expression group exhibited higher mortality rates (Fig. 1C). It is worth noting that miR-107 expression was not significantly correlated with patients' survival ( $\mathrm{P}=0.488 ; \mathrm{HR}=1.123$; 95\% CI: 0.598-2.117; Fig. S1A), while CDK8 expression was correlated with patients' survival $(\mathrm{P}=0.0167 ; \mathrm{HR}=2.376 ; 95 \%$ CI: 1.237-4.417; Fig. S1B).

UASRI and miR-107 interacted with each other but did not regulate the expression of each other. The interaction between UASR1 and miR-107 was predicted using IntaRNA2.0, which showed that UASR1 and miR-107 could form strong base pairing (Fig. 2A). The interaction between UASR1 and miR-107 was investigated by performing dual luciferase activity assay, which was performed by co-transfecting CR4 cells with UASR1 luciferase vector + miR-107 mimic (miR-107 group) or UASR1 luciferase vector + NC miRNA (NC group). Compared with the $\mathrm{NC}$ group, relative luciferase activity was significantly lower in miR-107 group (Fig. 2B; $\mathrm{P}<0.05)$. To further investigate the interaction between UASR1 and miR-107, CR4 cells were transfected with the UASR1 expression vector or miR-107-mimic. Overexpression of UASR1 and miR-107 was confirmed by RT-qPCR (Fig. 2C; $\mathrm{P}<0.05)$. Compared with the $\mathrm{NC}$ and $\mathrm{C}$ groups, overexpression of UASR1 and miR-107 did not significantly affect the expression of each other (Fig. 2D).

The expression of CDK8 was upregulated in CR4 cells with overexpression of UASR1. To test the possibility of UASR 1 as an internal sponge of miR-107, the effects of overexpression of UASR 1 and miR-107 on the expression of CDK8, a miR-107 target, were evaluated by RT-qPCR (Fig. 3A) and western blot analysis (Fig. 3B). Compared with the $\mathrm{C}$ group, overexpression of miR-107 led to the downregulated expression of CDK8 $(\mathrm{P}<0.05)$. Overexpression of UASR1 served an opposite role and decreased the effects of miR-107 overexpression $(\mathrm{P}<0.05)$.

UASR1 promoted CR4 cell proliferation through the miR-107/CDK8 axis. The effects of overexpression of 
A

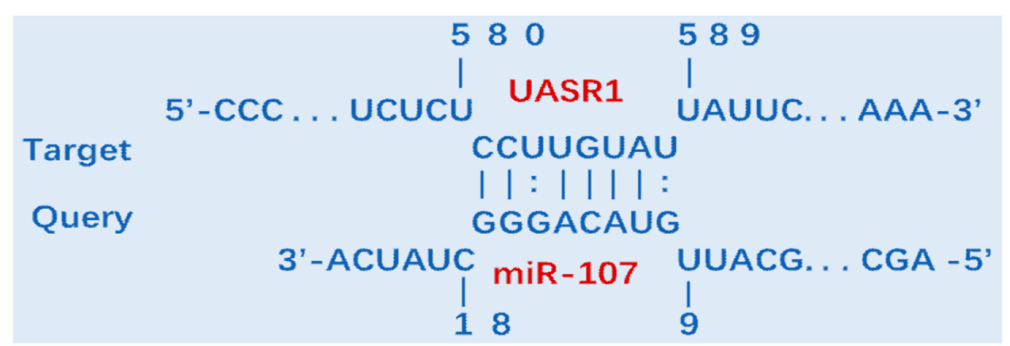

C
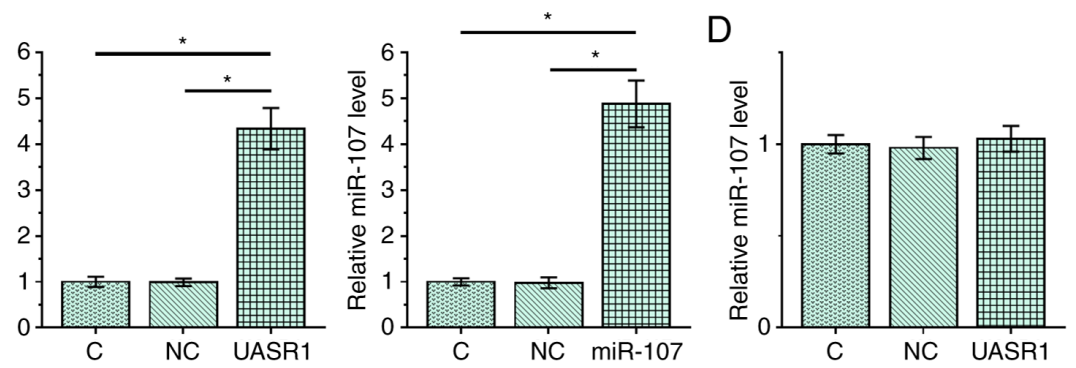
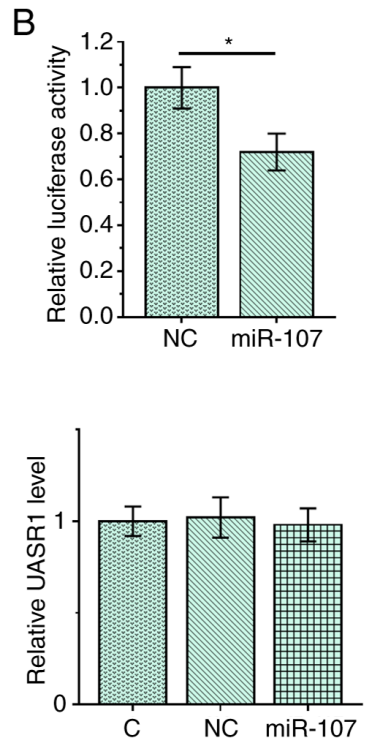

Figure 2. UASR1 and miR-107 interacted with each other but did not regulate the expression of each other. (A) The interaction between UASR1 and miR-107 was predicted using IntaRNA2.0. The interaction between UASR1 and miR-107 was investigated by performing a dual luciferase activity assay, which was performed by co-transfecting CR4 cells with UASR1 luciferase vector + miR-107 mimic (miR-107 group) or UASR1 luciferase vector + NC miRNA (NC group). (B) Luciferase activity was measured at $48 \mathrm{~h}$ post-transfection. To further investigate the interaction between UASR1 and miR-107, CR4 cells were transfected with the UASR1 expression vector or miR-107-mimic. (C) Overexpression of UASR1 and miR-107 was confirmed by performing RT-qPCR. (D) The effects of overexpression of UASR1 and miR-107 on the expression of each other were also analyzed by RT-qPCR. "P<0.05. RT-qPCR, reverse transcription-quantitative polymerase chain reaction; $\mathrm{NC}$, negative control.

A

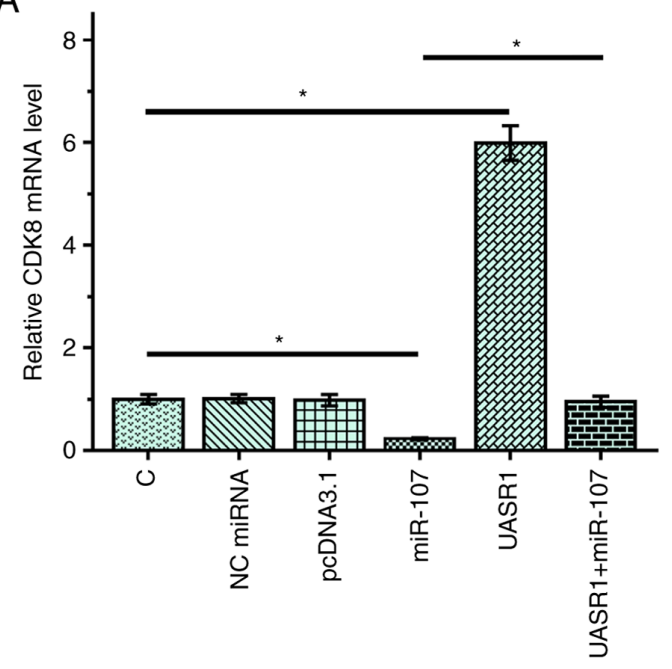

B
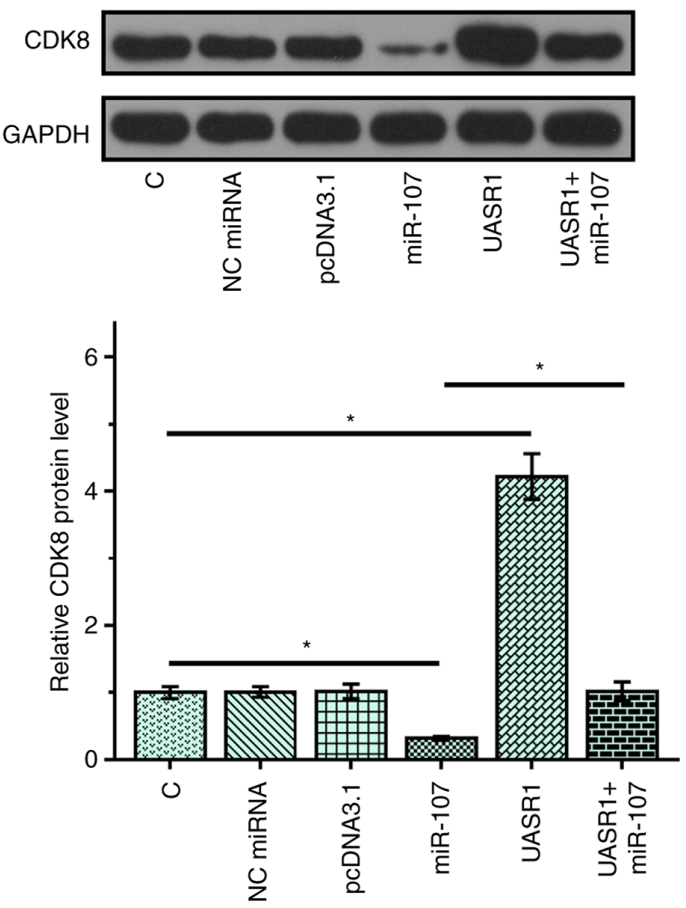

Figure 3. The expression of CDK8 was upregulated in CR4 cells with UASR1-overexpression. To test the possibility of UASR1 as an internal sponge of miR-107, the effects of overexpression of UASR1 and miR-107 on the expression of CDK8, a miR-107 target, were analyzed by (A) reverse transcription-quantitative polymerase chain reaction and (B) western blot analysis. All polymerase chain reaction reactions were repeated 3 times and mean values were presented and compared. ${ }^{\mathrm{P}}<0.05$. NC, negative control.

UASR1, miR-107 and CDK8 on the proliferation of CR4 cells were analyzed by performing cell proliferation. Overexpression of UASR 1 and CDK8 led to increased proliferation rate of $\mathrm{CR} 4$ cells $(\mathrm{P}<0.05)$. By contrast, overexpression of miR-107 led to decreased cell proliferation rate
$(\mathrm{P}<0.05)$. Furthermore, overexpression of UASR1 decreased the inhibitory effects of miR-107 on cell proliferation (Fig. 4A; $\mathrm{P}<0.05$ ). Overexpression of CDK8 in cells with CDK8 expression vector and the overexpression of UASR1 and miR-107 in cells co-transfected with UASR1 expression 
A

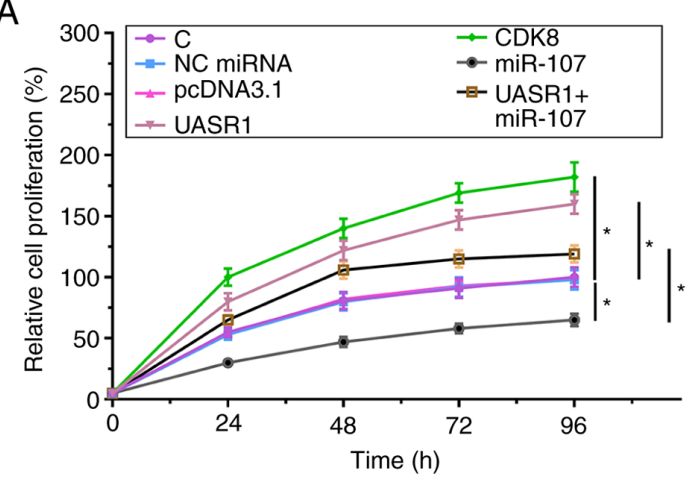

B

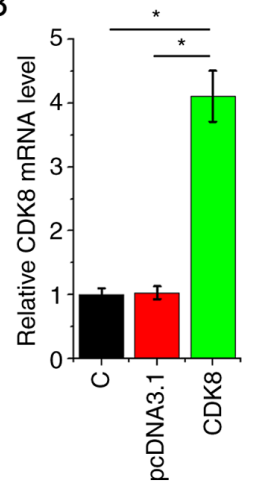

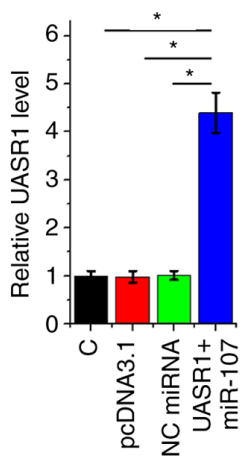

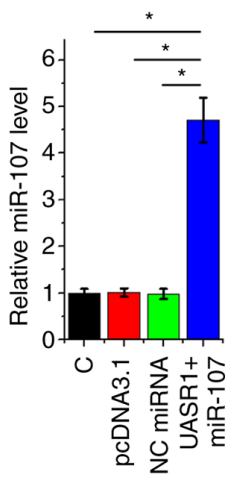

Figure 4. UASR1 promoted CR4 cell proliferation through the miR-107/CDK8 axis. (A) The effects of overexpression of UASR1, miR-107 and CDK8 on the proliferation of CR4 cells were analyzed by performing cell proliferation. (B) Reverse transcription-quantitative polymerase chain reaction was performed to confirm the overexpression of CDK8 in cells with the CDK8 expression vector and UASR1-overexpression and miR-107 in cells co-transfected with UASR1 expression vector and miR-107 mimic. ${ }^{*} \mathrm{P}<0.05$. NC negative control.

vector and miR-107 mimic were confirmed by RT-qPCR (Fig. 4B; $\mathrm{P}<0.05$ ).

\section{Discussion}

In the present study, the interactions between UASR1, miR-107 and CDK8 were studied in CRC. It was demonstrated that UASR1 was upregulated in CRC and that it may upregulate CDK8 by sponging miR-107, thereby promoting CRC cell proliferation.

The functionality of UASR1 has only been investigated in breast cancer (13). It was observed that UASR1 was upregulated in breast cancer and that it interacted with the AKT/mTOR pathway to promote the migration and proliferation of CRC cells. Another study reported that UASR1 was upregulated in CRC and that it interacted with the mTOR signaling pathway to promote cancer cell proliferation (14). The present study confirmed the upregulation of UASR 1 in CRC. In addition, increased proliferation of CRC cells was observed following overexpression of UASR1. Therefore, UASR 1 may serve an oncogenic role in CRC by promoting cancer cell proliferation.

Distant metastasis is common in patients with CRCs (18). Once distant metastasis has occurred, the prognosis will be extremely poor. The present study demonstrated that the high expression levels of UASR1 were closely correlated with the poor survival of patients with CRC. Therefore, measuring the expression levels of UASR1 prior to therapy may aid in the determination of treatment approaches, thereby improving the survival of patients with CRC. It is worth noting that high expression levels of CDK8 were also correlated with the poor survival of patients with CRC, while miR-107 expression had no significant effects on patient survival. The mechanism that correlates CDK8, but not miR-107, with patient survival remains to be investigated.

miR-107 serves tumor suppressive roles in several types of cancer $(15,19)$. However, the role of miR-107 in CRC is unknown. A recent study reported that miR-107 may target CDK8 to suppress the migration and proliferation of breast cancer cells (15). The present study showed that miR-107 also serves a tumor suppressive role in regulating CRC cell proliferation by targeting CDK8.
The present study showed that UASR1 and miR-107 interacted with each other in CRC cells. However, overexpression of miR-107 did not affect the expression of UASR1. Therefore, UASR1 is unlikely to be a target of miR-107. Notably, overexpression of UASR 1 decreased the inhibitory effects of overexpression of miR-107 on the expression of CDK8 and CRC cell proliferation. The results of the present study suggested that UASR1 is likely to be an internal sponge of miR-107.

It is known that miR-107 may also target CDK6 (20). However, in the CR4 CRC cell line used in the present study, miR-107-overexpression failed to significantly affect the expression of CDK6. Therefore, miR-107 may have different functions in different cells lines and/or different cancer types. It is worth noting that multiple CRC cell lines were used at the beginning. However, multiple cell transfections were included in the present study, and the CR4 cell line is the only cell line that showed satisfactory transfection efficiency of all the transfections.

The present study characterized a novel UASR $1 / \mathrm{miR}$ $107 /$ CDK8 pathway in CRC. However, the present study is limited by the small sample size. In addition, the in vivo interactions among UASR1, miR-107 and CDK8 have not been investigated. Future studies are required to include in vivo animal experiments and enroll more patients to further confirm these conclusions.

In conclusion, UASR1 is upregulated in CRC and was a predictor of poor survival. UASR1 may sponge miR-107 to upregulate $\mathrm{CDK} 8$, thereby promoting cancer cell proliferation.

\section{Acknowledgements}

Not applicable.

\section{Funding}

Not applicable.

\section{Availability of data and materials}

The datasets used and/or analyzed during the current study are available from the corresponding author upon reasonable request. 


\section{Authors' contributions}

QZZ performed the clinical studies, experiments work, data analysis and manuscript writing. ZSC performed the literature research, experiment design and manuscript revision. Both authors read and approved the final manuscript.

\section{Ethics approval and consent to participate}

The Ethics Committee of The Second Hospital of Shandong University approved the present study (approval no. CRC201233255). All the patients provided written informed consent.

\section{Patient consent for publication}

Not applicable.

\section{Competing interests}

The authors declare that they have no competing interests.

\section{References}

1. Siegel RL, Miller KD, Fedewa SA, Ahnen DJ, Meester RGS, Barzi A and Jemal A: Colorectal cancer statistics, 2017. CA Cancer J Clin 67: 177-193, 2017.

2. Bray F, Ferlay J, Soerjomataram I, Siegel RL, Torre LA and Jemal A: Global cancer statistics 2018: GLOBOCAN estimates of incidence and mortality worldwide for 36 cancers in 185 countries. CA Cancer J Clin 68: 394-424, 2018.

3. Favoriti P, Carbone G, Greco M, Pirozzi F, Pirozzi RE and Corcione F: Worldwide burden of colorectal cancer: A review. Updates Surg 68: 7-11, 2016.

4. Engstrand J, Nilsson H, Stromberg C, Jonas E and Freedman J: Colorectal cancer liver metastases-a population-based study on incidence, management and survival. BMC Cancer 18: 78, 2018.

5. Mahasneh A, Al-Shaheri F and Jamal E: Molecular biomarkers for an early diagnosis, effective treatment and prognosis of colorectal cancer: Current updates. Exp Mol Pathol 102: 475-483, 2017.

6. Symonds EL, Pedersen S, Cole SR, Massolino J, Byrne D, Guy J, Backhouse P, Fraser RJ, LaPointe L and Young GP: Improving participation in colorectal cancer screening: A randomised controlled trial of sequential offers of faecal then blood based non-invasive tests. Asian Pac J Cancer Prev 16: 8455-8460, 2015.
7. Tanaka S: Molecular pathogenesis and targeted therapy of pancreatic cancer. Ann Surg Oncol 23 (Suppl 2): S197-S205, 2016.

8. Li XL, Zhou J, Chen ZR and Chng WJ: P53 mutations in colorectal cancer-molecular pathogenesis and pharmacological reactivation. World J Gastroenterol 21: 84-93, 2015.

9. Heinemann V, Douillard JY, Ducreux M and Peeters M: Targeted therapy in metastatic colorectal cancer-an example of personalised medicine in action. Cancer Treat Rev 39: 592-601, 2013.

10. Wapinski $\mathrm{O}$ and Chang HY: Long noncoding RNAs and human disease. Trends Cell Biol 21: 354-361, 2011.

11. Lalevee S and Feil R: Long noncoding RNAs in human disease: Emerging mechanisms and therapeutic strategies. Epigenomics 7: 877-879, 2015.

12. Arun G, Diermeier SD and Spector DL: Therapeutic targeting of long non-coding RNAs in cancer. Trends Mol Med 24: 257-277, 2018.

13. Cao Z, Wu P, Su M, Ling H, Khoshaba R, Huang C, Gao H, Zhao Y, Chen J, Liao Q, et al: Long non-coding RNA UASR1 promotes proliferation and migration of breast cancer cells through the AKT/mTOR pathway. J Cancer 10: 2025-2034, 2019.

14. Wang W, Wang Z, Wang H, Li X and Wang HT: Promoting effect of PAX5-activated IncRNA UASR1 on growth of colorectal cancer by regulating the mTOR pathway. Eur Rev Med Pharmacol Sci 24: 2986-2993, 2020.

15. Li XY, Luo QF, Wei CK, Li DF, Li J and Fang L: miRNA-107 inhibits proliferation and migration by targeting CDK8 in breast cancer. Int J Clin Exp Med 7: 32-40, 2014.

16. Weiser MR: AJCC 8th edition: Colorectal cancer. Ann Surg Oncol 25: 1454-1455, 2018.

17. Livak KJ and Schmittgen TD: Analysis of relative gene expression data using real-time quantitative PCR and the 2(-Delta Delta C(T)) method. Methods 25: 402-408, 2001.

18. Benson AB III, Bekaii-Saab T, Chan E, Chen YJ, Choti MA, Cooper HS, Engstrom PF, Enzinger PC, Fakih MG, Fenton MJ, et al: Metastatic colon cancer, version 3.2013: Featured updates to the NCCN guidelines. J Natl Compr Canc Netw 11: 141-152, 2013.

19. Lu C, Xie Z and Peng Q: miRNA-107 enhances chemosensitivity to paclitaxel by targeting antiapoptotic factor Bcl-w in non small cell lung cancer. Am J Cancer Res 7: 1863-1873, 2017.

20. Chen L, Zhang R, Li P, Liu Y, Qin K, Fa ZQ, Liu YJ, Ke YQ and Jiang XD: P53-induced microRNA-107 inhibits proliferation of glioma cells and down-regulates the expression of CDK6 and Notch-2. Neurosci Lett 534: 327-332, 2013.

(i) $($ ) This work is licensed under a Creative Commons Attribution-NonCommercial-NoDerivatives 4.0 International (CC BY-NC-ND 4.0) License. 